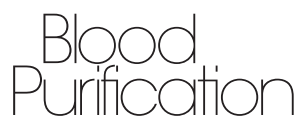

Amato, C. 77

Amerling, R. 36, 103

Audia, P. 103

Barros, S.P. 125

Beck, J.D. 125

Broumand, B. 39

Cazzavillan, S. 69, 106

Churchwell, M.D. 133

Corradi, V. 69, 106

Crawford-Bonadio, T.L. 48

Cruz, D. 69, 106

de Cal, M. 69, 106

DeOreo, P.B. 7

Diaz-Buxo, J.A. 48

Dubrow, A. 103

Feinfeld, D. 103
Fishbane, S. 53

Fissell, W.H. 12

Fleischman, A.J. 12

Gatti, E. 77

Gioberge, S. 77

Giordana, G. 77

Gotch, F. 18, 27, 139

Gruber, S. 103

Handelman, G.J. 58, 139

Harbord, N. 103

Hoenich, N.A. 62

Humes, H.D. 12

Kitzler, T. 27

Kotanko, P. 27, 139

Kshirsagar, A.V. 125

Kuhlmann, M.K. 120
Lazarus, J.M. 31

Lembcke, A. 115

Levin, N.W. 27, 69, 106, 139

Manfro, S. 106

Marcelli, D. 77

Moss, K.L. 125

Mueller, B.A. 133

National Kidney Foundation 112

Nelson, R.G. 112

Ocampo, C. 69

Offenbacher, S. 125

Ofsthun, N.J. 31

Polanco, N. 69

Rassu, M. 69, 106

Ratanarat, R. 69, 106
Ricci, Z. 106

Roessler, E. 106

Ronco, C. 36, 62, 69, 106

Roy, S. 12

Sands, J.J. 99

Sarkar, S.R. 27

Segala, C. 69, 106

Stopper, A. 77

Thijssen, S. 27

Tuttle, K.R. 112

Twardowski, Z.J. 90

Winchester, J.F. 36, 103

Wystrychowski, G. 27

Zhu, F. 27

\title{
Subject Index Vol. 25, No. 1, 2007
}

Anemia 31, 53

Arteriovenous fistula 99

- graft 99

Atherosclerosis, dental 125

Bacterial contamination 62

Body composition 27

Bundled payment 7

Calcification, coronary artery 115

Calcium mass balance, kinetic model 139

- scoring, coronary 115

Case mix adjustment 7

Central venous catheter 99

Chronic kidney disease 69, 112,133

Computed tomography, electron beam 115

Conflicts of interest 36

Continuous renal replacement therapy 106

Coronary artery 115
Database, dialysis 77

Diabetes 112

- mellitus 39

Diabetic nephropathy 39

Dialysis 12, 31, 139

- access 99

- - complications 99

- adequacy 48

- catheter 99

- duration 90

- fluid composition 62

-, quality of treatment 77

DNA, bacterial 106

End-stage renal disease 39, 53

Epidemiology, reverse 27

Erythropoietin 31

Extracellular fluid 139

Guidelines, KDOQI 112

Haemodialysis 62

Health insurers 36

Hemodiafiltration 120

Hemodialysis 18, 27, 58, 99
Hemoglobin 31, 53

Hyperphosphatemia, management 120

Hypertension 90

Hypotension, intradialytic 90

Imaging, coronary artery 115

Infection, subclinical 69

Inflammation 69

K/DOQI guidelines 2006103

Kidney disease 125

$\mathrm{Kt} / \mathrm{V}_{\text {urea }} 90$

Lag phenomenon 90

Maintenance hemodialysis 39

Medical economics 48

Medicare 31

- composite rate 7

Monitoring system 77

Mortality 18

Multi-slice spiral computed tomography 115

Nanotechnology 12

Oral pathogens 125
Oxalosis, systemic 58

Payer mix 7

Periodontal disease 125

Peritoneal dialysis 103

Pharmacokinetics 133

Pharmacotherapy 133

Phosphorus balance 120

Polytetrafluoroethylene graft 99

Practice guidelines 36

Renal failure, acute 106

- replacement 12

- - therapy 39

Sepsis 106

Serum antibody 125

Sodium profiling 90

Treatment time 18

Ultrafiltrate 106

Ultrafiltration 90

Uremic toxins 27

Vascular access 99

Vitamin C 58

\section{KARGER}

(c) 2007 S. Karger AG, Basel

Fax +41 613061234

E-Mail karger@karger.ch

www.karger.com 